\title{
Nivel de conocimiento de los pacientes con artritis reumatoide acerca de su enfermedad y tratamiento
}

\author{
Ana María Werner $C^{1}$, Sonia Kaliski $K^{1}$, Karina Salazar Q ${ }^{2 a}$, \\ Luis Bustos M ${ }^{3 b}$, María Teresa Rojas R ${ }^{3 c}$, Carlos Baumert L' ${ }^{3 c}$, \\ Hellmuth Leal L3d.
}

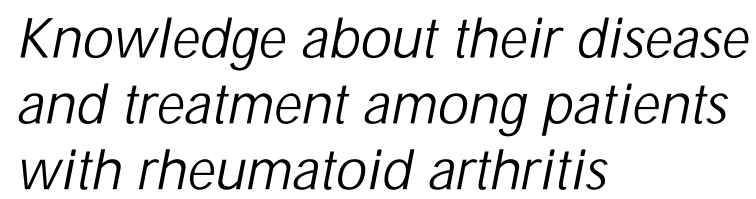

Background: The transfer of information in the physician-patient relationship is important, especially in chronic diseases such as Rheumatoid Arthritis (RA), since it influences the perception and behavior that the patient has about his or her illness. Aim: To evaluate the level of knowledge and concern of their illness and treatment and their relationship with functional disability, perception of the pain and global assessment of disease activity, in patients with RA. Patients and Methods: Cross sectional study of 104 patients (mean age 56 years, 100 women) with RA. Demographic and clinical variables were registered. The knowledge about their illness and requirement of further information and concern about aspects of the illness and treatment, were assessed. Physical functioning was measured using the Health Assessment Questionnaire (HAQ). A visual analogue (VAS) and Likert scales were used for a global assessment of disease activity. Results: Sixty percent of patients had only primary school studies. The median evolution of the illness was 14 years. Ninety eight percent knew their diagnosis and 91\% required further information. There was a high degree of concern about the disease and treatment. The average HAQ score was 0.9 . There was a statistically significant relationship between HAQ score and pain VAS ( $r=0.41, \mathrm{p}<0.01$ ). There was a moderate agreement between the global assessment of disease activity made by patients and physicians (Kappa $=0.499 ; \mathrm{p}=0.000$ ). Conclusions: Even though patients with RA are informed about their disease, they require further information. Their highest concern is about the functional consequences of RA and they perceive a higher activity of the disease than their treating physicians (Rev Méd Chile 2006; 134: 1500-06).

(Key words: Arthritis, Rheumatoid; Pain measurement; Visual analogue pain scale)

\author{
Recibido el 28 de junio, 2005. Aceptado el 14 de junio, 2006. \\ ${ }^{1}$ Servicio de Medicina Intema y Unidad de Reumatología Hospital Dr. Hemán Henníquez Aravena, \\ Temuco. Facultad de Medicina-Departamento de Medicina Intema, Universidad de La Frontera- \\ Temuco. ${ }^{2}$ Hospital Dr. Hemán Henńquez Aravena, Temuco. ${ }^{3}$ Universidad de La Frontera, Temuco. \\ aEnfermera Universitaria \\ bBioestadístico, CIGES \\ cAlumno de Medicina, Facultad de Medicina, Universidad de La Frontera \\ dDoctor en Ciencias Biológicas
}

Correspondencia a: Dra. Ana María Werner. Centro Médico SIRESA. Manuel Montt 942, 2º piso, Temuco. Teléfonos: 45960000, 45-738946. Fax: 45-325053. E mail: amwemer@sumet.cl 
$\mathrm{U}$ no de los aspectos importantes en la relación médico-paciente diaria, dice relación con la comunicación y transferencia de información. Esta transferencia es particularmente importante en pacientes con enfermedades crónicas como la artritis reumatoide (AR), donde un adecuado conocimiento de la enfermedad por parte del paciente, permite un cambio en la conducta de éste respecto de su enfermedad, cooperación y adherencia a tratamientos, en ocasiones complejos; y en este reconocimiento de la AR, cuyo curso es impredecible, el autocuidado resulta esencial ${ }^{1-3}$, debiendo el paciente ser capaz de aprender a aceptar e integrar la enfermedad como parte de su vida diaria ${ }^{4}$.

Los pacientes con AR desean conocer más acerca de su enfermedad, aunque sólo menos de la mitad recibe información de parte de los profesionales de la salud ${ }^{5}$. Muchos buscan la información fuera del ambiente clínico de salud. Se ha documentado que pacientes no reciben toda la información que desean ni toda aquella que necesitan ${ }^{6}$. Se menciona en la literatura diferentes programas de educación para pacientes con AR y sus resultados, algunos con beneficios sobre el dolor, sobre la incapacidad funcional, la evaluación global del paciente como también sobre su estado anímico ${ }^{7-}$ 12. Por tanto, se hace necesario conocer a nuestros pacientes con AR sus variables demográficas y qué nivel de conocimiento actual poseen de su enfermedad y tratamiento para ofrecerles el/los programa(s) de educación más adecuado(s) según nuestra realidad.

El propósito de este trabajo es evaluar el nivel de conocimiento y preocupación que tienen los pacientes con AR sobre su enfermedad y tratamiento y su relación con incapacidad funcional, percepción del dolor y evaluación global de la enfermedad, medida ésta simultáneamente por el paciente y el médico.

\section{PACIENTES Y MÉTODOS}

Estudio de corte transversal, realizado en una muestra no probabilística por conveniencia en 104 pacientes con AR, según los criterios ACR 198713, que consultan en policlínico de Reumatología del Hospital Dr. Hernán Henríquez Aravena de Temuco. Mediante cuestionario voluntario, se evaluó edad, sexo, procedencia, escolaridad, etnia, años de evolución, si conocían su diagnóstico y el tratamiento para la enfermedad que padecían, como también si requerían mayor información sobre ésta. Mediante Escala Análoga Visual (EVA) de 0-100 ( 0 =ninguno, $100=$ mucho), se evaluó qué conocimiento consideraban que tenían sobre su enfermedad y el grado de satisfacción de la información recibida. Con una escala ordinal de 5 niveles -Nada, Un poco, Bastante, Mucho y Muchísimo- se evaluó la preocupación por el dolor, deformidad articular, incapacidad funcional, inflamación articular; y respecto del tratamiento, se evaluó la preocupación por la duración, efectos secundarios y la efectividad de éste. Se aplicó el Cuestionario Evaluación Salud (HAQ) (0-3) para evaluar función física, EVA dolor paciente y se realizó evaluación global de la enfermedad por paciente y médico mediante la Escala Likert de 5 puntos -Muy bien, Bien, Regular, Mal y Muy mal-.

Estadística. Se utilizó el Programa Stata 8.0, análisis descriptivo a través de tablas estadísticas y medias de resumen, comparación de variables cualitativas, chi cuadrado o Fisher, Kappa y las cuantitativas, análisis de varianza, coeficiente de correlación de Pearson, con prueba t de Student para varianzas iguales y distintas. El nivel de significación para las pruebas estadísticas fue de $5 \%$.

\section{RESULTADOS}

A un total de 104 pacientes se les aplicó el cuestionario voluntario, 100 eran mujeres (96,2\%), con una media de edad de 56,2 años, de etnia mapuche, 18 pacientes (17,3\%). El 60,6\% de los pacientes evaluados tenían estudios de enseñanza básica. El 86,5\% eran de procedencia urbana y con una evolución media de la enfermedad de 13,9 años. Evaluada la función física mediante HAQ, se obtuvo un promedio de 0,9. La evaluación global de la enfermedad por el paciente y médico, para efectos del presente estudio, se categorizó en Muy bien-Bien, Regular, Mal-Muy mal. Se obtuvo 49\% para categoría Regular según paciente y 56,7\% para categoría Muy bien-Bien según el médico. Evaluación del dolor por el paciente mediante EVA se obtuvo como promedio $41 \mathrm{~mm}$ (Tabla 1). Respecto del grado de conoci- 
Tabla 1. Características demográficas y clínicas de los pacientes

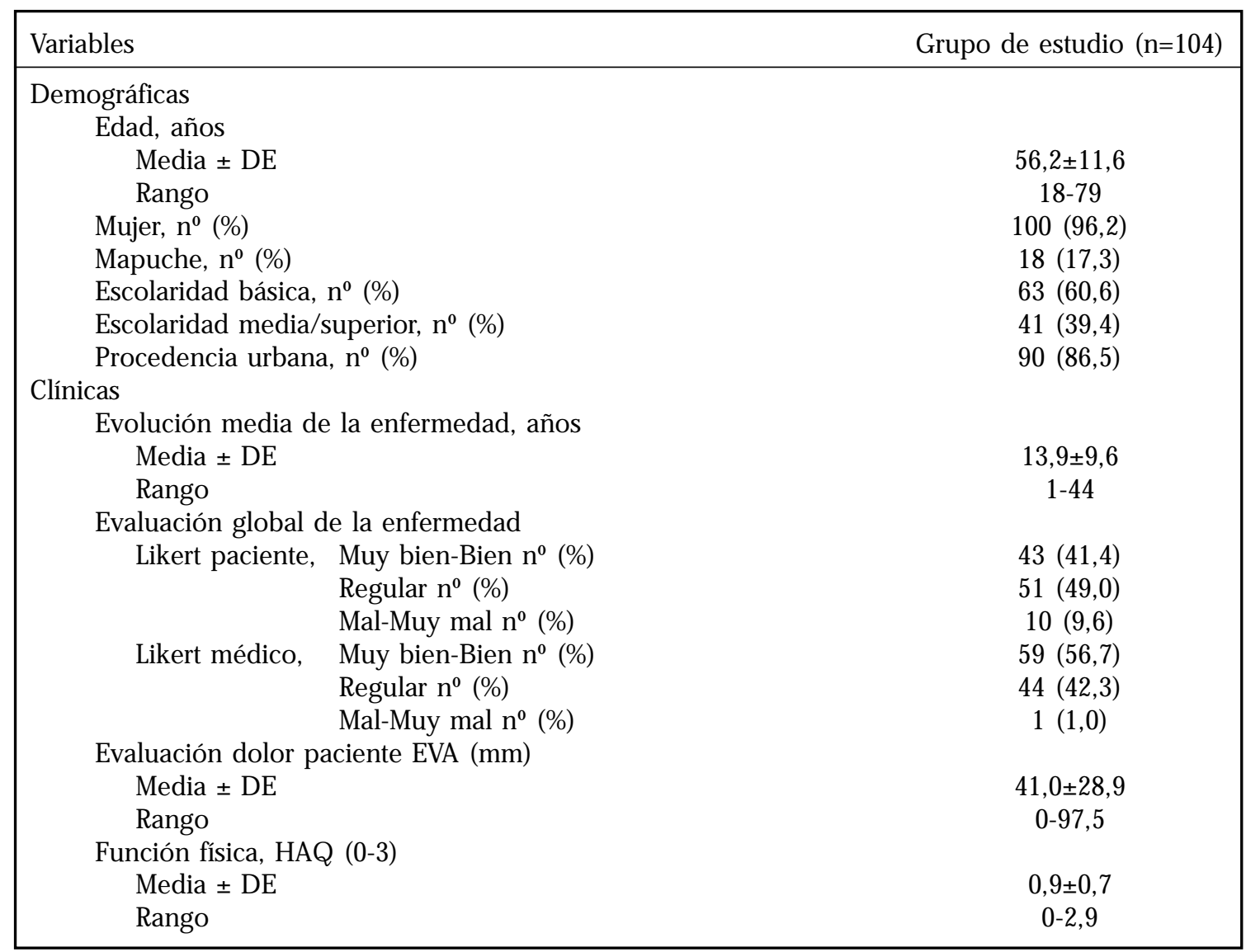

miento de los pacientes sobre su enfermedad y tratamiento, correspondió a $98,1 \%$ y $89,4 \%$, respectivamente. Estos fueron informados de su enfermedad por el reumatólogo en $67,3 \%$ de los casos. El 91,4\% indicó que requerían mayor información de su enfermedad, y 86\% de los pacientes encuestados les hubiera gustado recibir esta información de parte del reumatólogo. La información actual de su enfermedad correspondió a un EVA promedio de $44,5 \mathrm{~mm}$, y la satisfacción de la información recibida obtuvo un EVA promedio de 49,7 mm (Tabla 2).

El grado de preocupación que los pacientes manifestaban sobre el dolor, incapacidad funcional, deformidad e inflamación articular, así como algunos aspectos relacionados con el tratamiento como duración, efectos secundarios y eficacia son mostrados en la Tabla 3.
Para efectos de análisis de los resultados se agruparon las categorías en Nada-Un poco y Bastante-Mucho-Muchísimo. Observándose una gran preocupación por su enfermedad y tratamiento, destacando una mayor preocupación por la incapacidad funcional, la deformidad e inflamación articular que le puede provocar su AR.

El nivel educacional no influyó en la demanda de más información sobre su enfermedad, sin embargo, los pacientes con nivel de educación Medio-Superior tenían un valor de EVA promedio más elevado de conocimiento de su enfermedad ( $p=0,0002$ ) (Tabla 4).

La preocupación por la incapacidad funcional no se relacionó con un HAQ elevado ( $p=0,279)$ (Tabla 5). Se observó una relación estadísticamente significativa entre HAQ elevado y mayor años de evolución de la enfermedad ( $p=0,0031$; 
Tabla 2. Información del paciente sobre su enfermedad y tratamiento

\begin{tabular}{|c|c|}
\hline Variables & Grupo de estudio $(n=104)$ \\
\hline Conocía su enfermedad, no (\%) & $102(98,1)$ \\
\hline Conocía su tratamiento, no (\%) & $93(89,4)$ \\
\hline \multicolumn{2}{|l|}{ Información recibida } \\
\hline Reumatólogo no (\%) & $70(67,3)$ \\
\hline Médicos Generales nำ (\%) & $19(18,3)$ \\
\hline Otros profesionales de la salud no (\%) & $15(14,4)$ \\
\hline Requerían mayor información, no (\%) & $95(91,4)$ \\
\hline \multicolumn{2}{|l|}{ Información actual de su enfermedad } \\
\hline \multicolumn{2}{|l|}{ EVA mm } \\
\hline Media $\pm \mathrm{DE}$ & $44,5 \pm 26,9$ \\
\hline \multicolumn{2}{|l|}{ Satisfacción de la información EVA mm } \\
\hline Media $\pm \mathrm{DE}$ & $49,7 \pm 28,2$ \\
\hline
\end{tabular}

Tabla 3. Grado de preocupación de su enfermedad y tratamiento

\begin{tabular}{|c|c|c|}
\hline \multirow[b]{2}{*}{ Variables } & \multicolumn{2}{|c|}{ Grupo de estudio $(\mathrm{n}=104)$} \\
\hline & Nada-Un poco & Bastante-Mucho-Muchísimo \\
\hline Dolor, $\mathrm{n}^{\mathrm{o}}(\%)$ & $15(14,4)$ & $89(85,6)$ \\
\hline Incapacidad funcional, $\mathrm{n}$ - (\%) & $3(2,9)$ & $101(97,1)$ \\
\hline Deformidad articular, no (\%) & $5(4,8)$ & $99(95,2)$ \\
\hline Inflamación articular, no (\%) & $5(4,8)$ & $99(95,2)$ \\
\hline Duración del tratamiento, $\mathrm{n}$ - $(\%)$ & $31(29,8)$ & $73(70,2)$ \\
\hline Efectos secundarios, $\mathrm{n}$ o (\%) & $32(30,8)$ & $72(69,2)$ \\
\hline Eficacia, no (\%) & $16(15,4)$ & $88(84,6)$ \\
\hline
\end{tabular}

Tabla 4. Escolaridad y conocimiento sobre su enfermedad

\begin{tabular}{|lcccc|}
\hline & \multicolumn{4}{c|}{ Conocimiento enfermedad EVA (mm) } \\
Escolaridad & $\mathrm{n}$ - & Media & DE & $\mathrm{p}$ \\
\hline Básica & 63 & 37,2 & 28,0 & 0,0002 \\
Media-Superior & 41 & 55,7 & 20,8 & \\
Total & 104 & 44,5 & 26,9 & \\
\hline
\end{tabular}

t de Student para varianzas distintas.

Tabla 5. Preocupación por incapacidad funcional y H AQ

\begin{tabular}{|lrrrr|}
\hline Incapacidad funcional & $\mathrm{n}^{\mathbf{0}}$ & Media & DE & $\mathrm{p}$ \\
\hline Nada-Un poco & 3 & 0,4 & 0,4 & 0,279 \\
Bastante-Mucho-Muchísimo & 101 & 0,9 & 0,7 & \\
Total & 104 & 0,9 & 0,7 & \\
\hline
\end{tabular}

t de Student para varianzas iguales. 
Tabla 6. Efectividad del tratamiento y evaluación global de la enfermedad por el paciente

\begin{tabular}{|lrrrrrrr|}
\hline & \multicolumn{7}{c|}{ Evaluación global por el paciente } \\
Efectividad del tratamiento & Muy bien-Bien & \multicolumn{2}{c|}{ Regular } & Mal-Muy mal & \\
& $\mathrm{n}-$ & $\%$ & $\mathrm{n}^{\mathbf{0}}$ & $\%$ & $\mathrm{n} \underline{0}$ & $\%$ & $\mathrm{n}^{\mathbf{0}}$ \\
\hline Nada-Un poco & 5 & 15,2 & 21 & $63,6^{*}$ & 7 & 21,2 & 33 \\
Bastante-Muy efectivo-Excelente & 38 & $53,5^{*}$ & 30 & 42,3 & 3 & 4,2 & 71 \\
Total & 43 & 41,4 & 51 & 49,0 & 10 & 9,6 & 104 \\
\hline
\end{tabular}

Prueba exacta de Fisher.

$r=0,29)$ y entre HAQ elevado y EVA dolor paciente $(p=0,0000 ; r=0,41)$, aunque los índices de correlación fueron bajos.

Treinta y siete de los pacientes $(35,9 \%)$ consideraron su tratamiento actual como bastante efectivo», al agruparlo con los de la categoría muy efectivo» esto aumentó a 62 pacientes (60,2\%). Resultó significativo, que a mayor efectividad del tratamiento, la evaluación global de la enfermedad por el paciente puntuó en Bien-Muy bien ( $p$ $=0,000$ ) (Tabla 6).

Por otro lado, la evaluación global de la enfermedad realizada por el paciente y médico presentó una concordancia moderada con un Kappa de 0,499 (IC 95\% 0,44-0,56; $\mathrm{p}=0,000$ ).

\section{DiscUSIÓN}

Se acepta que la educación es un componente importante en el manejo de la AR y la falta de conocimiento exhibida por muchos pacientes, hace imprescindible la realización de programas de educación. Por ello es importante identificar los requerimientos educacionales de los pacientes con AR y su conocimiento básico de la enfermedad. Para lo anterior, se han desarrollado cuestionarios para evaluar el conocimiento que tienen los pacientes de las posibles causas, evolución, tratamiento de la AR y necesidad de mayor información de su enfermedad $5,14,15$. En este trabajo, no utilizamos los instrumentos de evaluación descritos en los estudios referenciados, sino más bien quisimos explorar si conocían el nombre de su enfermedad, su tratamiento y la percepción que nuestros pacientes tenían sobre el nivel de conocimiento de su patología, así como el grado de satisfacción sobre la información recibida.

Muchos pacientes están interesados en conocer más sobre su enfermedad ${ }^{16}$, y este interés parece no disminuir con el paso del tiempo. Sylvers et $\mathrm{al}^{15}$, encuestaron 101 pacientes con AR con un promedio de duración de la enfermedad de 14 años, indicando que $92 \%$ deseaba conocer más acerca de su enfermedad, muy similar a los resultados de nuestro estudio, considerando el paso de dos décadas.

Asimismo, nuestros pacientes mantienen su preocupación tanto por la enfermedad como por su tratamiento. Referente a este último aspecto, el interés de los pacientes está enfocado principalmente a su eficacia, lo que indicaría que éstos advierten que al ser más efectivo el tratamiento, la evaluación global de la enfermedad sería mejor.

Diferencia importante se observa respecto al estudio realizado por Cortés et al ${ }^{17}$, en el cual todos los pacientes estudiados conocían su enfermedad y su tratamiento, lo que les arrojó una menor preocupación por ambos, sugiriendo esto la importancia que tendría un paciente bien informado de su patología, así como el apoyo asistencial permanente del personal de salud involucrado en la atención reumatológica.

La mayor preocupación observada en nuestro grupo de pacientes con AR estudiados, fue la incapacidad funcional que la enfermedad le puede provocar, pero esto no se correlacionó con un HAQ elevado; similar resultado obtuvieron Cortés et $\mathrm{al}^{17}$. La preocupación por la incapacidad funcional no necesariamente indica que su función física (HAQ) esté deteriorada en el momento de la evaluación. En este estudio obtuvimos una mayor puntuación HAQ -que revela un mayor deterioro de su función física-, 
en aquellos pacientes con más años de evolución de su enfermedad, situación que es inherente a la propia $\mathrm{AR}$, en la cual muchos pacientes experimentan un curso crónico fluctuante a pesar del tratamiento, presentando también daño articular progresivo, deformidad e incapacidad funcional ${ }^{18}$. Interpretando esto, podríamos señalar que la preocupación por la incapacidad funcional observada en nuestro grupo de estudio, revelaría más bien la incertidumbre que este aspecto consultado les podría provocar en el futuro.

La percepción del dolor presentó valores extremos, con una percepción mayor del dolor en aquellos pacientes cuya puntuación de HAQ también fue mayor.

La evaluación global de la enfermedad realizada simultáneamente por el paciente y por el médico utilizando escala de Likert ${ }^{19}$, demostró que el paciente consideró su estado de actividad de la enfermedad peor que aquella calificada por el médico. Diferentes estudios sugieren que médico y paciente tendrían en cuenta aspectos distintos cuando tratan de evaluar la actividad de la enfermedad, por ello parece razonable incluir ambas en la evaluación clínica ${ }^{20}$. En nuestro estudio aplicamos concordancia a la evaluación global de la enfermedad, arrojando un grado de concordancia moderada.

Considerando que la AR es una patología reumatológica crónica, y por lo cual la interacción entre reumatólogo y paciente debería ser periódica, ésta es una condición que no siempre se cumple, por la imposibilidad de acceso a horas reumatológicas a una consulta con el especialista en forma oportuna. Por lo anterior, nuestros pacientes estudiados señalaron que quienes le

\section{REFERENCIAS}

1. KIRWAN JR Patient education in Rheumatoid Arthritis. Current Opinion in Rheumatology 1990; 2: 336-9.

2. TaAl E, Rasker JJ, Wiegman O. Patient education and self-management in the rheumatic diseases: A self-efficacy approach. Arthritis Care and Research 1996; 9: 229-38. informan acerca de su enfermedad, si bien en su mayoría fue el reumatólogo, un porcentaje no despreciable correspondió a médicos generales. De allí que, una medida educativa estratégica adecuada sería proporcionar a los médicos generales información actualizada sobre $\mathrm{AR}$, a través de cursos periódicos de actualización, de tal forma que el primer eslabón educativo sea el médico general, a través de información verbal y folletos simples confeccionados con la ayuda del reumatólogo, consistente en preguntas y respuestas sobre $\mathrm{AR}$, en un lenguaje comprensible para la realidad de nuestra población.

De tal modo que mientras el paciente espera acceder al reumatólogo, posea nociones básicas de su enfermedad, y ya una vez insertado en el policlínico de reumatología, en donde la imagen del reumatólogo es clave, puesto que la mayońa de nuestros pacientes desea obtener la información directamente del especialista, redundaremos sobre los aspectos básicos de la AR y principalmente sobre el curso de la enfermedad y sus complicaciones. Adicionalmente, con la ayuda de la enfermera de la unidad de reumatología, nos aseguramos un monitoreo efectivo sobre la buena adherencia al tratamiento, así como la derivación expedita, ante la aparición de eventuales reacciones adversas suscitadas.

Consideraremos las guías prácticas ${ }^{21,22}$, cuestionarios previos y posteriores a los programas de educación ${ }^{23}$, preferencias educacionales señaladas por pacientes con $\mathrm{AR}^{24}$, la experticia de ciertos pacientes como un recurso educacional valioso tanto para ellos mismos como para otros pacientes con $\mathrm{AR}^{25}$, así como las recomendaciones que reúnen las necesidades de información y educación de los pacientes con AR en la práctica clínica diaria ${ }^{26}$.

3. TAAL E, Rasker JJ, Wiegman O. Group education for Rheumatoid Arthritis patients. Semin Arthritis Rheum 1997; 6: 805-16.

4. GRIFFith J, CARR A. What is the impact of early Rheumatoid Arthritis on the individual. Best Pract Res Clin Rheumatol 2001; 15: 77-90.

5. KaY EA, PunchaK SS. Patient understanding of the causes and medical treatment of Rheumatoid Arthritis. Br J Rheumatol 1988; 27: 396-8. 
6. BarLow JH, Wright CC. Knowledge in patients with Rheumatoid Arthritis: A longer term follow-up of a randomized controlled study of patient education leaflets. Br J Rheumatol 1998; 37: 373-6.

7. Ruemsma RP, Taal E, Kirwan JR, Rasker JJ. Patient education programmes for adults with Rheumatoid Arthritis. Br Med J 2002; 325: 558-9.

8. Hennel SL, Brownsel C Dawson JK. Development, validation and use of a patient knowledge questionnaire (PKQ) for patients with early Rheumatoid Arthritis. Rheumatology 2004; 43: 467-71.

9. Minnock P, Fitzgerald O, Bresnihan B. Quality of life, social support, and knowledge of disease in women with Rheumatoid Arthritis. Arthritis Rheum 2003; 49: 221-7.

10. MEMEL DS, KIRWAN JR. General practitioners' knowledge of functional and social factors in patients with Reumatoid Arthritis. Health and Social Care in the Community 1999; 7: 387.

11. Lundroth Y, Brattstrom M, Be山man I, Ekestaf G, Olofsson Y, Strombeck B et al. A problem-based education program for patients with Rheumatoid Arthritis: evaluation after three and twelve months. Arthritis Care Res 1997; 10: 325-32.

12. Niedermann K, Fransen J, Knols R, Uebelhart D. Gap between short- and long-term effects of patient education in Rheumatoid Arhritis patients: a systematic review. Arthritis Rheum 2004; 51: 388-98.

13. Arnett FC, Edworthy SM, Bloch DA, McShane DJ, FRIES JF, COOPER NS ET AL. The American Rheumatism Association 1987 revised criteria for the classification of Rheumatoid Arthritis. Arthritis Rheum 1988; 31: 315-24.

14. HiL J, Bird HA, Hopkins R, Lawton C, Wright V. The development and use of a patient knowledge questionnaire in Rheumatoid Arthritis. $\mathrm{Br} \mathrm{J}$ Rheum 1991; 30: 45-9.

15. Silvers IJ, Hovell MF, Weisman MH, Muelier MR. Assessing physician/patient perceptions in Rheumatoid Arthritis. Arthritis Rheum 1985; 28: 300-7.

16. Neame R, Hammond A, Deighton C. Need for information and for involvement in decision making among patients with Rheumatoid Arthritis: a questionnaire survey. Arthritis Care Res 2005; 53: 249-55.

17. Cortés MA, Fernández S, Peiro M, Gel C, CoromiNAS H, LLobet JM ET al. Nivel de conocimiento de los pacientes con Artritis Reumatoide sobre su enfermedad y tratamiento. Rev Esp Reumatol 2003; 30: 266.

18. American College of Rheumatology Subcommittee on Rheumatoid Arthritis Guidelines. Guidelines for the management of Rheumatoid Arthritis. Arthritis Rheum 2002; 46: 328-46.

19. Felson DT, Anderson JJ, Boers M, Bombardier C, Chernoff M, Fried B et al. The American College of Rheumatology preliminary core set of disease activity measures for Rheumatoid Arthritis clinical trials. Arthritis Rheum 1993; 36: 729-40.

20. BatLe-Gualda E. Evaluación objetiva del enfermo en la práctica clínica. Rev Esp Reumatol 2002; 29: 78-81.

21. HIL J. A practical guide to patient education and information giving. Bailliere's Clin Rheumatol 1997; 11: 109-27.

22. Schrieber L, CoLey M. Patient education. Best Pract. Res Clin Rheumatol 2004; 18: 465-76.

23. Hennell SL, Brownsell C, Dawson JK. Development, validation and use of a patient knowledge questionnaire (PKQ) for patients with early Rheumatoid Arthritis. Rheumatology 2004; 43: 467-71.

24. BaRLOW JH, CULEN LA, ROwE IF. Educational preferences, psychological well-being and selfefficacy among people with Rheumatoid Arthritis. Patient Educ Couns 2002; 46: 11-9.

25. SwIFT TL, DiePpe PA. Using expert patients' narratives as an educational resource. Patient Educ Couns 2005; 57: 115-21.

26. Fautrel B, Pham T, Gossec L, Combe B, Fupo RM, GoupiLe P ET AL. Role and modalities of information and education in the management of patients with Rheumatoid Arthritis: development of recommendations for clinical practice based on published evidence and expert opinion. Joint Bone Spine 2005; 72: 163-70. 